\title{
A simplified prognostic scoring system for Fournier's gangrene
}

\begin{abstract}
Introduction: Fournier's gangrene is an acute necrotizing fasciitis affecting the perineal, perianal regions and genitalia. The cornerstones of treatment Fournier's gangrene are urgent necrotic tissue debridement, broad-spectrum antibiotics and resuscitation. Despite advanced management policies, mortality from Fournier's gangrene is still high. The aim of this study was to present our experience in lowvolume general hospital in the management of Fournier's gangrene according to our advocated scoring system.
\end{abstract}

Patients and methods: A total of 68 patients were classified according to age, body mass index, early detection, area involved and comorbidity. The author advocated an eight- scale simplified prognostic scoring system with a maximum score of eighteen points denoting the highest risk of mortality and a minimum score of eight points carrying a relatively lower risk of mortality. The primary end point of the study was disease-related death and the secondary end point was length of hospital stay.

Results: There were three grades according out simplified prognostic scoring system; grade I from 8-10 points, grade II from 11-14 points and grade III from 15-18 points. Patients with grade I carried a lower mortality rate and less hospital stay than those with grade II and grade III.

Conclusion: We tried to develop a reliable tool to predict severity of the disease, not only to identify patients at highest risk of major complications or death but also to provide a target for medical teams and researchers aiming to improve outcome and to collect beneficial information for proper management of patients with Fournier's gangrene.

Keywords: Fournier's gangrene, scoring system, simplified
Volume I Issue 3 - 2014

\author{
Aly Saber,' Tahir M Bajwa ${ }^{2}$ \\ 'Consultant Surgeon, Port-Fouad General Hospital, Egypt \\ ${ }^{2}$ Consultant surgeon, Services hospital, Pakistan
}

Correspondence: Aly Saber, Consultant Surgeon, Department of General Surgery, Port-Fouad General Hospital, Port-Fouad, Port-Said, Egypt, Tel +20I223752032, Fax +20663400848, Email alysaber54@gmail.com

Received: October 22, 2014 | Published: December 13, 2014
Abbreviations: FG, Fournier's gangrene; BMI, body mass index; LRINEC, laboratory risk indicator for necrotizing fasciitis; FGSI, Fournier's gangrene severity index; UFGSI, uludag Fournier's gangrene severity index

\section{Introduction}

Fournier's gangrene (FG) is an acute progressive infective necrotizing fasciitis affecting mainly the perineal, perianal regions and external genitalia of men, but can also occur in women and children. ${ }^{1}$ Fournier's gangrene was considered as an idiopathic syndrome but in the majority of cases, urogenital and perineal traumas, including pelvic and perineal injury or pelvic interventions, are incriminated. ${ }^{2}$ Systemic comorbidities are also identified in patients with Fournier's gangrene such as diabetes mellitus (DM), malignancy and malnutrition. ${ }^{3}$ The cornerstones of treating patients with Fournier's gangrene are urgent necrotic tissue debridement, proper doses of broad-spectrum antibiotics and resuscitation with fluids. ${ }^{1}$ Despite advanced management policies, mortality from Fournier's gangrene is still high. ${ }^{1,4}$ The aim of this study was to present our experience in low-volume general hospital in the management of Fournier's gangrene according to our advocated scoring system.

\section{Patients and methods}

A total of 68 patients presented with manifestations of Fournier's gangrene were enrolled to the study from April 2000 to September
2013. Those patients were classified according to their age, body mass index (BMI), early detection, area involved whether single or multiple and comorbidity. According to this classification, the author adopted a simplified prognostic scoring system for prediction of mortality rate in his patients. Disease detection was considered early or delayed according to clinical and laboratory data. Medical comorbidity was traced as diabetes mellitus only or concomitant with other disease such as cardiovascular, renal or hepatic disease. Laboratory risk indicator for necrotizing fasciitis (LRINEC) was done for all patients upon admission. The parameters of this laboratory risk indicator are glucose level, C-reactive protein level, total leucocytic count, serum sodium, creatinine level, hemoglobin.

Early patient presentation was considered when there was localized pain, minor skin manifestations such as redness, hotness and crepitus but without apparent gangrene. Delayed presentation was noticed with body temperature above $38^{\circ} \mathrm{C}$, rapid pulse, offensive wound discharge and cutaneous wound gangrene. Late presentation was considered with systemic manifestations of shock. The local ethics committee had approved all operative procedures. Ethical approval for this study was granted by the ethical review committee under supervision of the general director of Port- Fouad general hospital, Port-Fouad, PortSaid, Egypt.

\section{The simplified prognostic scoring system}

Here, the author advocated an eight-scale simplified prognostic 
scoring system with a maximum score of eighteen points denoting the highest risk of mortality and a minimum score of eight points carrying a relatively lower risk of mortality.

\section{Patient's age:}

$\leq 50$ years $=$ I point. $\geq 50$ years $=2$ points.

II. BMI:

$\mathrm{BMI}<25=$ I point. $\mathrm{BMI}<30=2$ points $\mathrm{BMI}>30=3$ points.

III. Temperature:

$<38^{\circ} \mathrm{C}=$ I point. $>38^{\circ} \mathrm{C}=2$ points.

IV. Pulse:

$<100$ beats/min=I point. $>100$ beats/min 2 points.

V. Systolic blood pressure:

$>90 \mathrm{~mm} \mathrm{Hg}=$ I point. $<90 \mathrm{~mm} \mathrm{Hg}=2$ points.

VI. Presentation:

Early $=1$ point. Delayed $=2$ points. Late $=3$ points.

VII. Area involved:

Single $=1$ point. multiple $=2$ points.

VIII.Comorbidity:

$\mathrm{DM}=1$ point. multiple $=2$ points.

\section{Study sites and technique}

Surgical interventions were performed in Port-Fouad general hospital, Port-Fouad, Port-Said, Egypt. Surgical interventions were of triple multimodal approach including hemodynamic stabilization, broad spectrum antibiotics, and surgical debridement. All necrotic and non-viable tissues were excised until the viable tissue was reached. Multiple sittings of surgical debridement were needed for the vast majority of cases. Very close patients' observation and wound care were mandatory.

\section{End points}

The primary end point of the study was disease-related death and the secondary end point was length of hospital stay.

\section{Results}

Patients were subdivided according to their ages and body mass indices as previously reported. The number of patients in each subgroup was traced as shown in (Table 1). Regarding the time of presentation, in vast majority of cases patients $(82.6 \%)$ presented with delayed and late courses of the disease and only 12 patients $(17.45 \%)$ presented with early disease manifestations according to (Table 2).

The extent of the disease was determined as single primary site (Figure 1), two or multiple sites including the primary sites and disease extension sites (Figure 2). Genitalia were the most common primary site of the disease in men (Figure 3), where gluteal region was the most common in women where there was no gluteal region involvement as a primary site in male patients of our series. Table 3 showed the number of patients regarding the primary site of the disease.
Table I Showed patients distribution regarding their ages and BMIs

\begin{tabular}{lllll}
\hline \multirow{2}{*}{ Item } & \multirow{4}{*}{ Subgroup } & \multicolumn{3}{l}{ Number } \\
\cline { 3 - 5 } & & Male & Female & Total \\
\hline \multirow{2}{*}{ Age } & $\leq 50$ years & 14 & 6 & 20 \\
& $\leq 50$ years & 28 & 20 & 48 \\
& BMI $<25$ & 24 & - & 24 \\
BMI & BMI $<30$ & 12 & 10 & 22 \\
& BMI $>30$ & 6 & 16 & 22 \\
\hline
\end{tabular}

Table 2 Showed patients distribution regarding time of their presentations

\begin{tabular}{llllll}
\hline \multirow{2}{*}{ Item } & \multicolumn{4}{l}{ Age subgroup } & \multicolumn{2}{l}{ BMI subgroup } \\
\cline { 2 - 6 } & $\begin{array}{l}\mathbf{5} \mathbf{5 0} \\
\text { years }\end{array}$ & $\begin{array}{l}>\mathbf{5 0} \\
\text { years }\end{array}$ & BMI<25 & BMI<30 & BMI>30 \\
\hline Early & 8 & 4 & 7 & 3 & 2 \\
Delayed & 6 & 20 & 7 & 9 & 10 \\
Late & 6 & 24 & 10 & 10 & 10 \\
Total & 20 & 48 & 24 & 22 & 22 \\
\hline
\end{tabular}

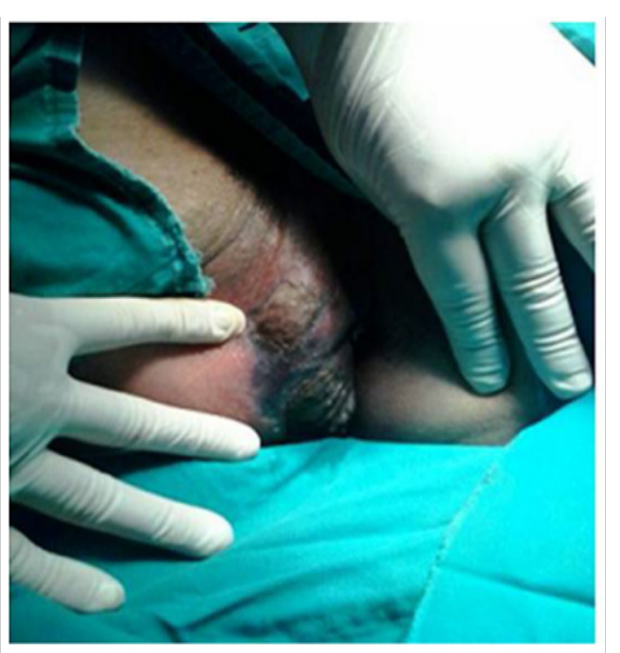

Figure I A pretreatment photograph showing the perianal involvement as single primary site.

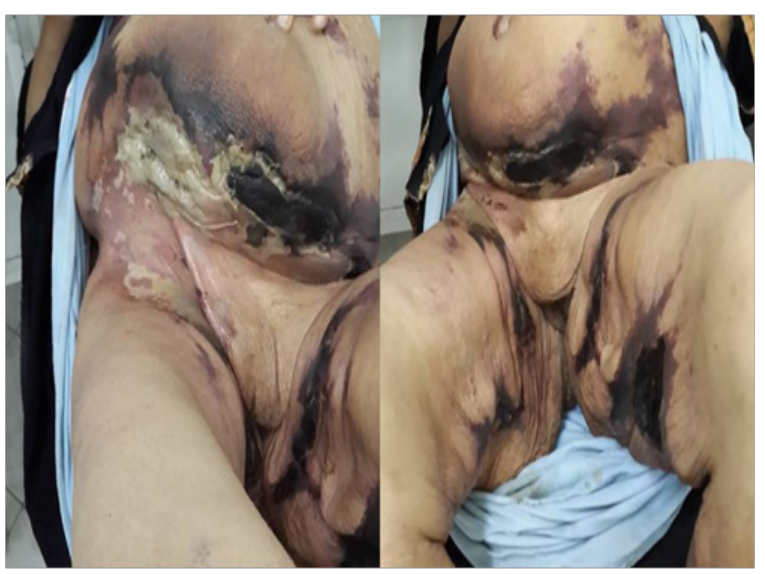

Figure 2 A photograph showing the genitalia as the primary site and disease extension to the lower abdominal wall in a woman. 


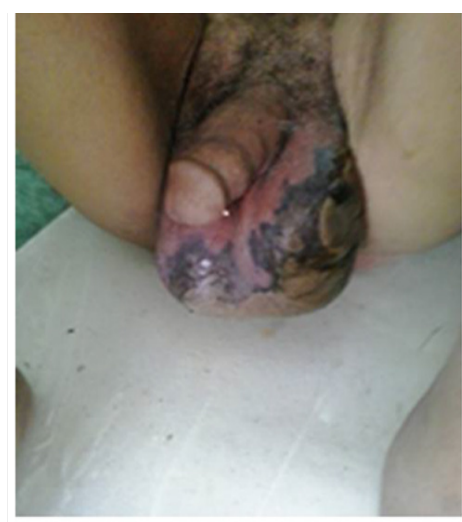

Figure $3 \mathrm{~A}$ photograph showing genitalia were the most common primary site of the disease in men.

Table 3 Showed patients regarding the primary site of the disease

\begin{tabular}{llll}
\hline Site & Male & Female & Total \\
\hline Genitalia & 20 & 8 & 28 \\
Perianal & 9 & 4 & 13 \\
Perineal & 13 & 4 & 17 \\
Gluteal & - & 10 & 10 \\
Total & 42 & 26 & 68
\end{tabular}

In the present study, 28 patients $(41.1 \%)$ died as a direct result of the disease. Age above 50years, higher BMI and delayed presentation were associated with much more incidence of mortality as shown in Table 4 . The mortality rate was higher in females $(69 \%)$ as we noticed that 18 of 26 female patients died as a result of the disease. In case of male patients, the mortality rate was $28.6 \%$ as a total of 12 of 42 patients died as a result of the disease. Regarding the adopted simplified prognostic scoring system, there were three grades; grade I from 8-10 points, grade II from 11-14 points and grade III from 1518 points. Patients with score of 8-10 points [grade I] carried a lower mortality rate and less hospital stay than those with score of 11-14 points [grade II] and score of 15-18 points [grade III]. The twelve patients who presented at the early stage were belonged to grade I score while delayed and late presentations were evident with grade II and grade III scores. The simplified prognostic scoring system was directly proportional to the mortality rate where patients with higher grades [grades II and III] showed more mortality compared with those having grade I score.

Table 4 Showed mortality in relation to patient's age, BMI and presentation

\begin{tabular}{llll}
\hline Factor & & Male [ $\mathbf{N}=12]$ & Female $\mathbf{N}=18$ \\
\hline \multirow{2}{*}{ Age } & $\leq 50$ years & 2 & - \\
& $\leq 50$ years & 10 & 18 \\
& BMI $<25$ & 1 & 2 \\
BMI & BMI $<30$ & 6 & 8 \\
& BMI $>30$ & 5 & 8 \\
Presentation & Early & - & - \\
& Delayed & 5 & 8 \\
& Late & 7 & 10
\end{tabular}

The hospital stay was calculated as the time of admission, resuscitation, surgical interference and time needed for wound care until patient was discharged. The time of hospital stay ranged from 7 to 30days which was 7-10days in case of early presented patients with grade I score while patients presented with delayed or late course of the disease and required both grades II and III, showed much more hospital stay as 15-30days. As regard to hospital stay, our simplified prognostic scoring system was directly proportional to the hospital stay where patients with higher grades [grades II and III] showed more hospital stay compared with those having grade I score.

\section{Discussion}

Fournier's gangrene is a progressive and fulminant necrotizing fasciitis of the genital, perianal and perineal regions that may extend to the abdominal wall between the fascial planes. ${ }^{5}$ There are two important validated scoring systems for outcome prediction of Fournier's gangrene. These systems are Fournier's Gangrene Severity Index (FGSI) and Uludag Fournier's Gangrene Severity Index (UFGSI). ${ }^{6}$ FGSI score uses nine parameters including the body temperature, heart rate, respiratory rate, hematocrit, white blood cell count, and serum levels of sodium, potassium, creatinine and bicarbonate. ${ }^{7}$ Yilmazlar et al., ${ }^{8}$ suggested a new scoring system, the Uludag FGSI (UFGSI), adding the age and the extent of the disease scores to the FGSI.

Although FGSI score is higher in non survivors, the difference was found statistically insignificant and some researchers concluded that the FGSI had no prognostic value ${ }^{4,9}$ and some other studies have shown no relationship between high FGSI scores and mortality. ${ }^{10}$ Conversely, some studies revealed that FGSI scores were sensitive and specific for predicting mortality rate ${ }^{11}$ and on comparing UFGSI against FGSI, it was concluded that despite including more variables, the UFGSI does not seem to be more powerful than the FGSI. ${ }^{12}$ Both scoring systems lack the timing of patient presentation, body mass index (BMI) and patient's comorbidity. ${ }^{6-12}$

The present study advocated an eight- scale simplified prognostic scoring system with a maximum score of eighteen points denoting the highest risk of mortality and a minimum score of eight points carrying a relatively lower risk of mortality. The proposed system contains patient's age, BMI, temperature, pulse, systolic blood pressure, timing of presentation, area involved and comorbidity. Many studies found obesity, ${ }^{13,14}$ the timing of patient presentation and patient's comorbidity as important parameters for outcome prediction of Fournier's gangrene and is considered as a major risk factor for mortality. ${ }^{1,3,5,15,16}$

Mortality rate in patients with Fournier's gangrene is usually high and depends on many related risk factors according the already validated scoring systems or our proposed simplified system. In the present study the overall mortality rate was $41.1 \%$ with $69 \%$ in females and $28.6 \%$ in males. In studies of same interest, the overall mortality rate was $25-30 \%{ }^{16,17}$ and may reach up to $88 \%{ }^{18}$ while was higher in females than males in series of Czymek et al. ${ }^{19}$ Higher mortality rate is usually detected with increasing age $>50$ years and the time interval between the first symptom and surgical intervention. ${ }^{1,3,5,18}$ Our results came in agreement with these reports and others of same interest.

The extension of the disease beyond the primary site and the mortality rate are controversial themes in the literature. Some studies have reported that the spread of the disease is related to a higher death 
rate, while others reported that the extension of the gangrene does not relate to a poorer prognosis. ${ }^{5}$ It was found that the extension of the infection to the abdominal wall ${ }^{5}$ and thighs ${ }^{20}$ is a predictor of mortality.

The associated medical illnesses included diabetes, chronic renal failure, chronic liver disease; hypertension, alcoholism, and peripheral vascular disorder usually carry the higher rates of mortality. ${ }^{16}$ Other researchers reported that various co-morbidities are known to be associated with FG, of which diabetes is the most common but its association with increased mortality is controversial. ${ }^{21,22}$ In most series, the majority of the patients had $\mathrm{DM}^{4,23}$ and the percentage of their occurrence varied from $54 \%{ }^{15}$ to $70 \% .{ }^{1}$ Existence of one or more of such co-morbidities as DM, alcoholism, neurological diseases, malignancy, immunosuppression, and, liver and kidney diseases can affect morbidity and mortality rates in cases with FG. DM is the most common predisposing factor with an incidence of $46-76.9 \%$ and mortality has been reported in $36-50 \%$ of these patients. ${ }^{22}$

\section{Conclusion}

Fournier's gangrene, although rare, is usually a potentially lethal disease. Co-morbidities, especially diabetes mellitus increase the mortality rate. Repeated surgical excisions and debridement with broad spectrum antibiotic provide good results. We tried to develop a reliable tool to predict severity of the disease, not only to identify patients at highest risk of major complications or death but also to provide a target for medical teams and researchers aiming to improve outcome and to collect beneficial information for proper management of patients with Fournier's gangrene.

\section{Sources of funding}

The corresponding author declares he has neither competing interest nor funding sources.

\section{Acknowledgements}

None.

\section{Conflict of interest}

The author declares no conflict of interest.

\section{References}

1. Mallikarjuna MN, Vijayakumar A, Patil VS, et al. Fournier's Gangrene: Current Practices. ISRN Surg. 2012:942437.

2. Zermani R, Bocchi F. A case of Fournier's gangrene: an insidious and dangerous pathology. Acta Biomed. 2012;83(3):217-219.

3. Ugwumba FO, Nnabugwu II, Ozoemena OF. Fournier's gangreneanalysis of management and outcome in south-eastern Nigeria. S Afr J Surg. 2012;50(1):16-19.

4. Aridogan IA, Izol V, Abat D, et al. Epidemiological characteristics of Fournier's gangrene: a report of 71 patients. Urol Int. 2012;89(4):457461.

5. Benjelloun el B, Souiki T, Yakla N, et al. Fournier's gangrene: our experience with 50 patients and analysis of factors affecting mortality. World J Emerg Surg. 2013;8(1):13.
6. Tuncel A, Keten T, Aslan Y, et al. Comparison of different scoring systems for outcome prediction in patients with Fournier's gangrene:Experience with 50 patients. Scand J Urol. 2014;48(4):393-399.

7. Laor E, Palmer LS, Tolia BM, et al. Outcome prediction in patients with Fournier's gangrene. J Urol. 1995;154(1):89-92.

8. Yilmazlar T, Ozturk E, Ozguc H, et al. Fournier's gangrene: an analysis of 80 patients and a novel scoring system. Tech Coloproctol. 2010;14(3):217-223.

9. Lujan Marco S, Budia A, Di Capua C, et al. Evaluation of a severity score to predict the prognosis of Fournier's gangrene. BJU Int. 2010;106(3):373-376.

10. Tuncel A, Aydin O, Tekdogan U, et al. Fournier's gangrene: three years of experience with 20 patients and validity of the Fournier's gangrene severity index score. Eur Urol. 2006;50(4):838-843.

11. Corcoran AT, Smaldone MC, Gibbons EP, et al. Validation of the Fournier's gangrene severity index in a large contemporary series. $J$ Urol. 2008;180(3):944-948.

12. Roghmann F, von Bodman C, Loppenberg B, et al. Noldus J Is there a need for the Fournier's gangrene severity index? Comparison of scoring systems for outcome prediction in patients with Fournier's gangrene. BJU Int. 2012;110(9):1359-1365.

13. Wang L, Han X, Liu M, et al. Experience in management of Fournier's gangrene: a report of 24 cases. J Huazhong Univ Sci Technolog Med Sci. 2012;32(5):719-723.

14. Kara E, Muezzinoglu T, Temeltas G, et al. Evaluation of risk factors and severity of a life threatening surgical emergency: Fournier's gangrene (a report of 15 cases). Acta Chir Belg. 2009;109(2):191-197.

15. Verma S, Sayana A, Kala S, et al. Evaluation of the Utility of the Fournier's Gangrene Severity Index in the Management of Fournier's Gangrene in North India:A Multicentre Retrospective Study. J Cutan Aesthet Surg. 2012;5(4):273-276.

16. Jeong HJ, Park SC, Seo IY, et al. Prognostic factors in Fournier gangrene. Int J Urol. 2005;12(12):1041-1044.

17. Eke N. Fournier's gangrene. A review of 1726 cases. Br J Surg. 2000;87(6):718-728.

18. Sorensen MD, Krieger JN, Rivara FP, et al. Fournier's gangrene: population based epidemiology and outcomes. JUrol. 2009;181(5):2120 2126.

19. Czymek R, Frank P, Limmer S, et al. Fournier's gangrene: is the female gender a risk factor? Langenbecks Arch Surg. 2010;395(2):173-180.

20. Vyas HG, Kumar A, Bhandari V, et al. Prospective evaluation of risk factors for mortality in patients of Fournier's gangrene: A single center experience. Indian J Urol. 2013;29(3):161-165.

21. Ersoz F, Sari S, Arikan S, et al. Factors affecting mortality in Fournier's gangrene: Experience with fifty-two patients. Singapore Med J. 2012;53(8):537-540.

22. Unalp HR, Kamer E, Derici H, et al. Fournier's gangrene: Evaluation of 68 patients and analysis of prognostic variables. J Postgrad Med. 2008;54(2):102-105.

23. Sroczynski M, Sebastian M, Rudnicki J, et al. A complex approach to the treatment of Fournier's gangrene. Adv Clin Exp Med. 2013;22(1):131135 